\title{
Molecular hydrogen line emission from the reflection nebula Parsamyan 18
}

\author{
S. D. Ryder, ${ }^{\star} \dagger$ L. E. Allen, M. G. Burton, M. C. B. Ashley and J. W. V. Storey \\ School of Physics, University of New South Wales, Sydney 2052, Australia
}

Accepted 1997 October 7. Received 1997 August 19; in original form 1997 June 10

\begin{abstract}
ABS TR ACT
The newly commissioned University of New South Wales Infrared Fabry-Perot (UNSWIRF) has been used to image molecular hydrogen emission at 2.12 and $2.25 \mu \mathrm{m}$ in the reflection nebula Parsamyan 18. P 18 is known to exhibit low values of the $(1-0) /(2-1) S(1)$ ratio, suggestive of UV-pumped fluorescence rather than thermal excitation by shocks. Our line ratio mapping reveals the full extent of this fluorescent emission from extended arc-like features, as well as a more concentrated thermal component in regions closer to the central exciting star. We show that the emission morphology, line fluxes and gas density are consistent with the predictions of photodissociation region (PDR) theory. Those regions with the highest intrinsic $1-0 \mathrm{~S}(1)$ intensities also tend to show the highest $(1-0) /(2-1) S(1)$ line ratios. Furthermore, variations in the line ratio can be attributed to intrinsic fluctuations in the incident radiation field and/or the gas density, through the self-shielding action of $\mathrm{H}_{2}$. An isolated knot of emission discovered just outside $\mathrm{P} 18$, and having both an unusually high $(1-0) /(2-1) \mathrm{S}(1)$ ratio and relative velocity, provides additional evidence for an outflow source associated with P 18.
\end{abstract}

Key words: molecular processes - shock waves - ISM: individual: Parsamyan 18 ISM: molecules - reflection nebulae.

\section{INTRODUCTION}

The 'cometary nebula' Parsamyan 18 (P 18= NGC $2316=\mathrm{L} 1654)$ has properties characteristic of both reflection and emission nebulae. Near-infrared emission lines of molecular hydrogen have been observed, as well as emission features at 3.3, 6.2, 7.7, 8.6 and $11.3 \mu \mathrm{m}$ (Sellgren 1986; Burton et al. 1990; Sellgren, Werner \& Allamandola 1996) attributed to aromatic hydrocarbons. Similar emission features and near-infrared colours were found in over half of the visual reflection nebulae surveyed by Sellgren et al. (1996). López et al. (1988) used a combination of optical, infrared and radio continuum observations to argue that the two brightest regions of $\mathrm{P} 18$ are not in fact stars, but actually represent light from a heavily obscured $\mathrm{H}$ II region breaking through a dust shell surrounding the exciting early B-type star. On the basis of (unpublished) optical spectra, however, Sellgren et al. list both components as being stel-

^E-mail: sryder@jach.hawaii.edu

$\dagger$ Present address: Joint Astronomy Centre, 660 N. A’Ohoku Place, Hilo, HI 96720, USA. lar, with the north-eastern star (star 'A', a B2-3e star with $V=13.21$ ) illuminating the dust as well as exciting the atomic and molecular hydrogen.

P 18 was only the second object (after NGC 2023; Gatley et al. 1987) found to have infrared $\mathrm{H}_{2}$ emission-line ratios similar to the predictions of UV-pumped fluorescence, rather than thermal excitation by shocks (Sellgren 1986). In nearly all such studies to date, line ratios and inferred excitation mechanisms are based on large-aperture spectra, or from only a single pointing. With the advent of imaging infrared Fabry-Perot systems (with resolving powers $R \gtrsim 1000$ that provide the necessary contrast between line and continuum), it has now become possible to carry out line ratio mapping. This could be a useful aid in discriminating between the sources of excitation, on the basis of line ratio trends and morphology. In this paper we report the results of our $\mathrm{H}_{2}$ line imaging of $\mathrm{P} 18$ using the University of New South Wales Infrared Fabry-Perot (UNSWIRF).

Our results show that 'collisional fluorescence' is occurring in $\mathrm{P} 18$. That is, pure fluorescent line ratios are thermally modified by the effects of collisions. This occurs in dense molecular clouds where $\mathrm{H}_{2}$ self-shielding maintains a 
significant column density of the molecule close to the cloud surface (i.e. $A_{V}<1$ ), where the gas temperature can reach a few thousand degrees (e.g. Sternberg \& Dalgarno 1989; Burton, Hollenbach \& Tielens 1990, hereafter BHT). Emission-line ratios are then sensitive to the density and radiation field, and so observations with sufficiently high spatial resolution can be used to trace the variation of these parameters within a source. Large-aperture measurements of both $\mathrm{H}_{2}$ and high- $J$ CO lines (e.g. Hayashi et al. 1985; Stutzki et al. 1990) of many such 'photodissociation regions' (PDRs) (Tielens \& Hollenbach 1985; BHT) have indicated they are clumpy, containing regions where the density must be 10-100 times greater than the surrounding cloud. High spatial resolution $\mathrm{H}_{2}$ line ratio measurements may now be used to probe how this high-density gas is distributed.

\section{OBSERVATIONS}

UNSWIRF is a 70-mm-diameter Queensgate (UK) Ltd etalon with $R \sim 4000$, tunable over both the $H$ and $K$ windows (Ryder et al. 1998, in preparation). When used in conjunction with the wide-field mode of IRIS ${ }^{1}$ at the $f / 36$ focus of the Anglo-Australian Telescope (AAT), a (roughly circular) field of 1.7-arcmin diameter at $0.77 \operatorname{arcsec}$ pixel $^{-1}$ is produced. Appropriate 1 per cent bandpass filters are used to ensure that only a single order is passed by the etalon.

Observations of $\mathrm{P} 18$ in the $\mathrm{H}_{2} 1-0 \mathrm{~S}(1)(2.122 \mu \mathrm{m})$ and $\mathrm{H}_{2} 2-1 \mathrm{~S}(1)(2.248 \mu \mathrm{m})$ lines were obtained on 1996 April 4 UT. A sequence of four images spanning the peak of the 2.122- $\mu \mathrm{m}$ line (as determined from observations of OMC-1) and separated by $\sim 2 / 3$ of the instrumental profile width were obtained with UNSWIRF, while for the 2.248 - $\mu$ m line, just three images spaced $\sim 1 / 2$ of the profile width apart were taken. In each case, another image at 3-4 profile widths from the line centre was obtained in order to sample the continuum. An integration time of $120 \mathrm{~s}$ per etalon setting was used, with non-destructive readouts every $5 \mathrm{~s}$. The same exposure sequence was carried out on the sky 5 arcmin east of P 18. The object and sky exposures were repeated (with slight offsets from the previous positions), leading to total on-source integration times of 1920 and $2880 \mathrm{~s}$ in the 2.122- and 2.248- $\mu \mathrm{m}$ lines, respectively.

Dark current subtraction and linearization are performed during readout. The object frames were sky-subtracted, and then flat-fielded using normalized dome flat-fields, using in each case images at the matching etalon settings. All frames were first registered using field stars, and all frames at a given etalon setting were averaged together. The continuum frames, appropriately scaled, were then subtracted from all other images to leave just pure line emission in each.

At this stage, the emission-line images were 'stacked' into cubes of increasing etalon spacing/wavelength, so that a Lorentzian profile could be fitted to the ( 3 or 4 ) point spectrum at each pixel. Having already fixed the continuum level to be zero, the number of free parameters in the fitting was further reduced by constraining the width of the profile to be the same as that found by fits to high-resolution scans of krypton arc lines (2.1165 and 2.2485 $\mu \mathrm{m}$ for the 2.12- and

${ }^{1}$ The Infrared Imager and Spectrometer (Allen et al. 1993) uses a $128 \times 128 \mathrm{HgCdTe}$ array manufactured by Rockwell International Science Centre, CA.
2.25- $\mu \mathrm{m}$ cubes, respectively). We justify this on the basis that the narrow $\mathrm{H}_{2}$ linewidths $\left(16-20 \mathrm{~km} \mathrm{~s}^{-1}\right)$ observed in P 18 by Burton et al. (1990) would not be resolvable by UNSWIRF (FWHM $\sim 65 \mathrm{~km} \mathrm{~s}^{-1}$ at $2.12 \mu \mathrm{m}$ ). The output of the line fitting includes maps of the peak intensity, as well as the etalon setting corresponding to that peak. Blanking masks are constructed by requiring that the fitted peak position must lie within the range covered by the etalon sequence. Flux calibration, and continuum scaling factors, are provided by photometry of $10 \times 1 \mathrm{~s}$ observations of the spectroscopic standard BS $2882(K=5.18)$, also at the same etalon settings as used for P 18.

\section{RESULTS}

Fig. 1 shows a grey-scale map of the $2.12-\mu \mathrm{m}$ line intensity, with contours of the three brightest $2.12-\mu \mathrm{m}$ continuum sources superimposed (including star 'A') to aid in comparing our new data to that elsewhere in the literature (e.g. López et al. 1988). The $\mathrm{H}_{2}$ emission structure can be conveniently divided into a number of distinct regions, as outlined in Fig. 2. The emission-line fluxes of each region are tabulated in Table 1. The integrated emission-line flux of P 18 measured from the $2.12-\mu \mathrm{m}$ image is $(13.1 \pm 0.6) \times 10^{-13} \mathrm{erg} \mathrm{\textrm {cm } ^ { - 2 }} \mathrm{s}^{-1}$, compared with the $(4.5 \pm 0.3) \times 10^{-13} \mathrm{erg} \mathrm{cm}^{-2} \mathrm{~s}^{-1}$ found in the matching $2.25-\mu \mathrm{m}$ image. Uncertainties in the absolute flux calibration and line-fitting procedures are roughly comparable in magnitude with the noise contribution $\left(\sim 1.8 \times 10^{-16} \mathrm{erg}\right.$ $\mathrm{cm}^{-2} \mathrm{~s}^{-1}$ ) per pixel over which the emission is summed.

The morphology of the infrared molecular hydrogen emission differs somewhat from the 'cometary' nature displayed in the optical (Cohen 1974), and from the broadband infrared and radio continuum images of López et al. (1988). Instead of a cone, the appearance is one of two incomplete, but overlapping, arcs. With reference to Fig. 2, one arc is outlined by the contiguous regions (1,2, 5 and 4), while the other is defined by the regions 4, 3, 6 and 7. Each arc, if it were part of a complete ring, would have a diameter of $\sim 30$ arcsec, or $0.16 \mathrm{pc}$, assuming P 18 to be $1.1 \mathrm{kpc}$ away (Hilton \& Lahulla 1995). Star 'A', assumed to be the exciting source, sits on the periphery of the arc that extends to the west, and halfway between the centre and the edge of the eastern arc. The emisssion peaks (in both the 2.12- and the $2.25-\mu \mathrm{m}$ images) in the areas due north and south of star ' $A$ ', where these two arcs overlap. In addition, more prominent emission at $2.12 \mu \mathrm{m}$ is found on the western edge of the eastern arc, $\sim 8 \operatorname{arcsec}$ due west of star 'A', as well as in an isolated knot of emission some 50 arcsec to the north-west of star 'A'. This isolated patch (hereafter designated P 18NW) has no continuum counterpart or obvious exciting source. Although none of this emission-line structure is apparent in the $K$-band image of López et al. (1988), it is faintly visible in the FITS version of the $K^{\prime}$ imaging survey of CO molecular outflow sources carried out by Hodapp (1994).

Fig. 2 is a grey-scale image of the $(1-0) /(2-1)$ ratio in $\mathrm{P} 18$ for all points in which the 2-1 intensity could be determined. The values found range from 1-2 in the eastern arc, to as much as 7 in the nebular arc just west of star 'A', and even higher in $\mathrm{P} 18-\mathrm{NW}$ (although the $2-1$ detection here is barely at the $1 \sigma$ level). Fig. 2 also demarcates the eight 


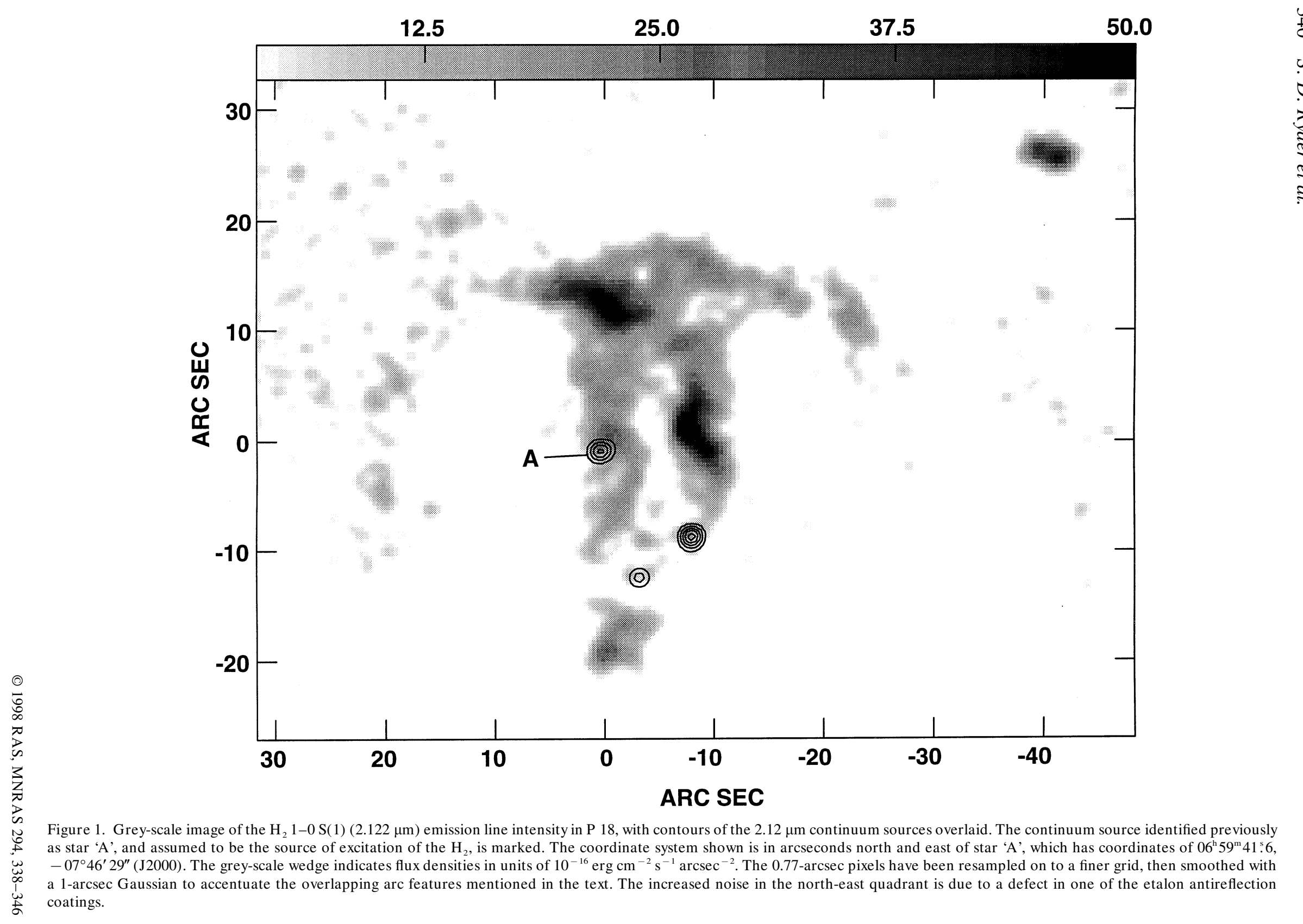




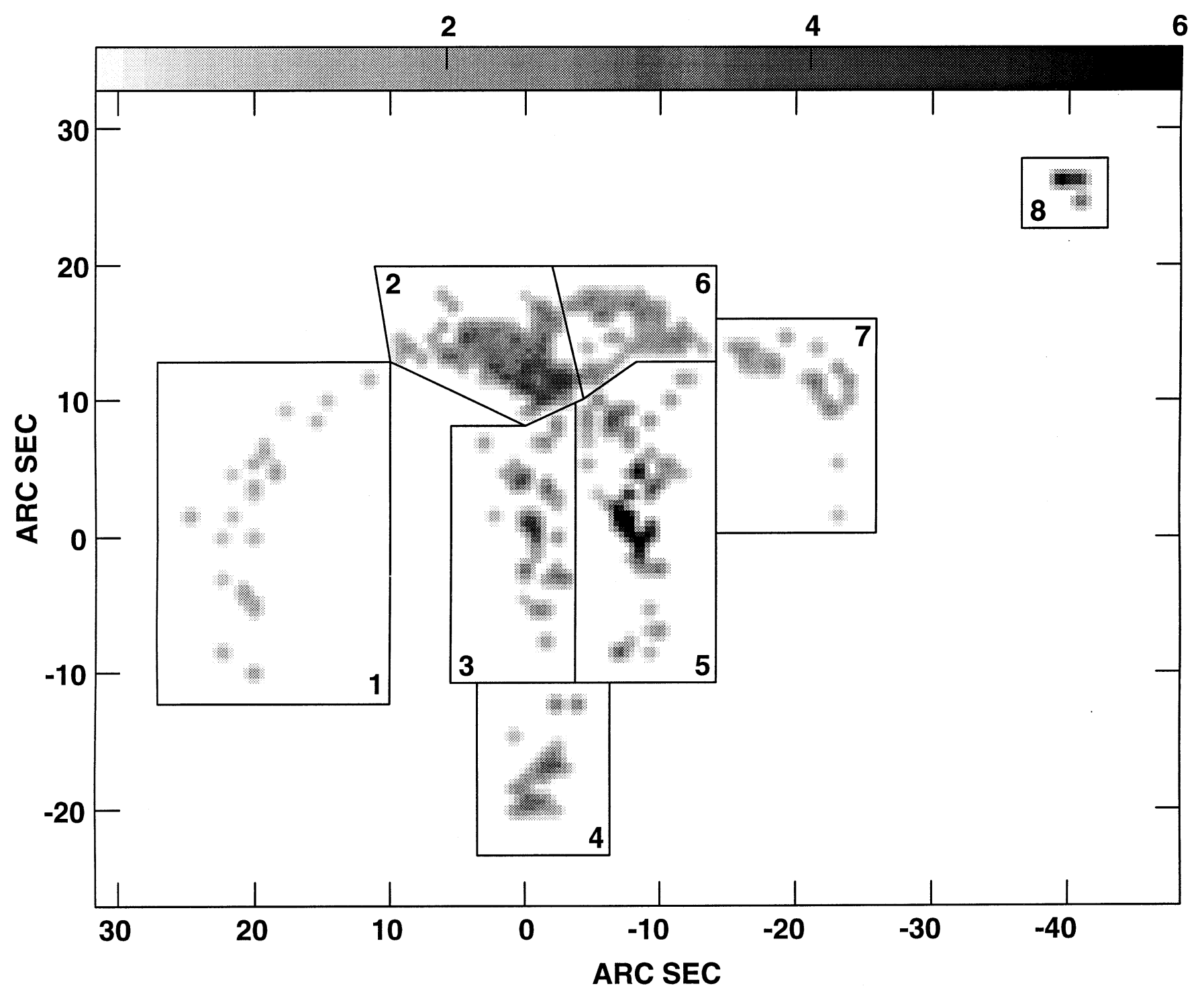

Figure 2. Grey-scale image of the $\mathrm{H}_{2} 1-0 \mathrm{~S}(1) / 2-1 \mathrm{~S}(1)$ ratio in $\mathrm{P} 18$, for all points in which a reliable detection ( $\mathrm{S} / \mathrm{N}>3$ ) at $2.25 \mu \mathrm{m}$ was achieved. The numbered polygons outline the eight separate emission regions in and around P 18, as given in Table 1. As in Fig. 1, the 0.77 -arcsec pixels have been resampled on to a finer grid, but no smoothing has been applied. 
Table 1. $\mathrm{H}_{2}$ line intensities, ratios and velocities in $\mathrm{P} 18$.

\begin{tabular}{|c|c|c|c|c|c|c|c|c|c|}
\hline Region $^{a}$ & $\begin{array}{r}d^{b} \\
(\operatorname{arcsec})\end{array}$ & $\begin{array}{r}\text { FUV } \\
\text { Field }^{c} \\
\left(\mathrm{G}_{0}\right)\end{array}$ & $\begin{array}{c}1-0 S(1) \\
\text { Flux } \\
\left(\operatorname{ergs~cm}^{-2} \mathrm{~s}^{-1}\right)\end{array}$ & $\begin{array}{c}2-1 S(1) \\
\text { Flux } \\
\left(\mathrm{ergs} \mathrm{cm}^{-2} \mathrm{~s}^{-1}\right)\end{array}$ & $\mathrm{N}^{d}$ & Ratio & $\begin{array}{c}\text { Peak }(1-0) S(1) \\
\text { Flux in region } \\
\left(\text { ergs cm }^{-2} \mathrm{~s}^{-1} \operatorname{arcsec}^{-2}\right)\end{array}$ & $\begin{array}{l}\text { Ratio } \\
\text { at Peak }\end{array}$ & $\begin{array}{r}\text { Relative } \\
\text { Velocity }^{e} \\
\left(\mathrm{~km} \mathrm{~s}^{-1}\right)\end{array}$ \\
\hline Whole Source & $\ldots$ & .. & $13.1 \pm 0.6(-13)$ & $4.5 \pm 0.3(-13)$ & 2147 & $2.9 \pm 0.4$ & & & $\ldots$ \\
\hline 1 (E Arc) & 22 & 2400 & $2.7 \pm 0.1(-13)$ & $1.1 \pm 0.1(-13)$ & 766 & $2.4 \pm 0.3$ & $1.7(-15)$ & 1.6 & +8 \\
\hline 2 (N Peak) & 15 & 5060 & $2.2 \pm 0.1(-13)$ & $8.1 \pm 0.5(-14)$ & 248 & $2.7 \pm 0.3$ & $5.5(-15)$ & 2.9 & +2 \\
\hline 3 (Central Arc) & $\ldots$ & $\ldots$ & $1.9 \pm 0.1(-13)$ & $4.9 \pm 0.4(-14)$ & 260 & $3.9 \pm 0.5$ & $3.1(-15)$ & 4.8 & 0 \\
\hline 4 (S Peak) & 18 & 3500 & $8.5 \pm 0.5(-14)$ & $3.3 \pm 0.3(-14)$ & 147 & $2.6 \pm 0.4$ & $3.1(-15)$ & 3.3 & -2 \\
\hline 5 (W Peak) & 8 & 17800 & $3.0 \pm 0.1(-13)$ & $8.3 \pm 0.6(-14)$ & 373 & $3.6 \pm 0.4$ & $7.9(-15)$ & 7.0 & -1 \\
\hline 6 (NW Arc) & 19 & 3200 & $1.1 \pm 0.1(-13)$ & $4.6 \pm 0.3(-14)$ & 141 & $2.5 \pm 0.3$ & $2.5(-15)$ & 2.9 & +4 \\
\hline 7 (W Arc) & 25 & 1800 & $9.7 \pm 0.5(-14)$ & $4.0 \pm 0.3(-14)$ & 172 & $2.4 \pm 0.3$ & $2.0(-15)$ & 2.4 & -3 \\
\hline $8(\mathrm{P} 18-\mathrm{NW})^{f}$ & 48 & 500 & $3.8 \pm 0.2(-14)$ & $4.4 \pm 1.2(-15)$ & 40 & $8.6 \pm 2.9$ & $4.9(-15)$ & 13.7 & +14 \\
\hline
\end{tabular}

${ }^{a}$ Numbering as in Fig. 2.

${ }^{b}$ Projected distance from star A.

${ }^{c}$ In units of the average interstellar field, for the projected distance from star A.

${ }^{d}$ Number of pixels, of size $0.77 \times 0.77 \mathrm{arcsec}^{2}$, included in region.

${ }^{e}$ Relative velocity of $\mathrm{H}_{2}$ emission from region.

${ }^{f}$ This region is not linked with the others, and is probably shock-excited rather than irradiated by star A (Section 4.4).

distinct regions whose fluxes, flux ratios and relative velocities are tabulated in Table 1.

\section{DISCUSSION}

\subsection{Fluorescent molecular hydrogen}

The average ratio of the $1-0$ and $2-1 \mathrm{~S}(1)$ lines over the whole of P 18 is $2.9 \pm 0.4$ (Table 1 ), as expected for fluorescently excited gas slightly modified by a thermal contribution to the $v=1-0 \mathrm{~S}(1)$ line. Pure fluorescent emission produces a line ratio of $\sim 1.7$ (e.g. Black \& Dalgarno 1976; Black \& van Dishoeck 1987), but this ratio can be exceeded when some of the gas is hot enough for the lower levels to be thermalized. We thus conclude, as did Sellgren (1986), that molecular gas in P 18 is irradiated by far-UV photons. It is situated in two arcs around star 'A', where some of the gas is hot enough for the $v=1$ level to be collisionally populated. This is characteristic of a dense photodissociation region (PDR) (Sternberg \& Dalgarno 1989; BHT). In these PDRs the gas is sufficiently dense that self-shielding of $\mathrm{H}_{2}$ occurs for optical depths $<1$ from the cloud surface, so that molecules can exist in this warm, primarily atomic region. The typical 1-0 S(1) line flux density in $\mathrm{P} 18$ is $\sim 4 \times 10^{-15} \mathrm{erg} \mathrm{cm}^{-2} \mathrm{~s}^{-1} \operatorname{arcsec}^{-2}$, and peaks at $8 \times 10^{-15} \mathrm{erg} \mathrm{cm}^{-2} \mathrm{~s}^{-1} \operatorname{arcsec}^{-2}$ just west of star 'A'. These fluxes are consistent with the predictions for PDR models for gas of average density, $n \sim 10^{4} \mathrm{~cm}^{-3}$, irradiated by farUV photons from star 'A', as we show below.

We estimate the far-UV flux from star 'A', a B2-3e star at $1.1 \mathrm{kpc}$ (Sellgren et al. 1996) to be $5060 G_{0}$ in a shell 15 arcsec away. Here $G_{0}=1$ represents the average interstellar radiation field $\left(=1.6 \times 10^{-3} \mathrm{erg} \mathrm{cm}^{-2} \mathrm{~s}^{-1}\right.$; Habing 1968). For gas of density $\sim 10^{4} \mathrm{~cm}^{-3}$ exposed to far-UV fields, $G_{0} \sim 10^{3-5}$, BHT predict $1-0$ S(1) line fluxes of $\sim 3 \times 10^{-15} \mathrm{erg} \mathrm{cm}^{-2} \mathrm{~s}^{-1} \operatorname{arcsec}^{-2}$ for a face-on PDR, consistent with the data. Note that our discovery of these arcs, and our interpretation of them as PDRs irradiated by star 'A', is at odds with the conclusion of López et al. (1988) that the continuum sources are not stellar, and merely unobscured sight-lines towards a compact $\mathrm{H}$ in region. While P 18 shares some of the properties of an $\mathrm{H}$ II region, we feel it would be too much of a coincidence if one of these spots also had a location and spectrum consistent with being the excitation source of the PDR.

For such conditions the models predict a purely fluorescent $(1-0) /(2-1) \mathrm{S}(1)$ line ratio of $\sim 1.7$, whereas the average ratio for the source is 2.9 . This suggests that about half the 1-0 S(1) emission is thermally contributed, and we discuss the implications of this further in Section 4.3.

\subsection{Geometry and kinematics}

The morphology suggests that star ' $A$ ' is surrounded by an (incomplete) shell seen in projection, and in this section we investigate whether the distribution of the $\mathrm{H}_{2}$ line emission is consistent with such a geometry.

Consider a spherical molecular shell surrounding an illuminating star as representing the arc through the north, west and south peaks. Assuming that the average density in the cloud is low enough that the emission is mainly fluorescent (see Section 4.3), the $\mathrm{H}_{2}$ emits primarily from a sheath whose thickness is $A_{V} \sim 1$, with dust extinction determining the depth to which the far-UV photons penetrate. Viewed from afar, the relative intensity along a line of sight is then proportional to the length of the sightline through the shell. We examined models where the thickness of the shell is varied as a proportion of its radius (see Fig. 3), and found that when this is $\sim 25$ per cent a reasonable fit to the data is achieved. Given the source distance of $1.1 \mathrm{kpc}$, this provides a physical size to the shell and, assuming a standard extinction curve [i.e. $A_{V}=1 \equiv N\left(\mathrm{H}_{2}\right)=2 \times 10^{21} \mathrm{~cm}^{-2}$ ], yields an average density; specifically, for the north, south and west peaks respectively, we obtain thicknesses of $\sim 9,5$ and $5 \times 10^{16} \mathrm{~cm}$ for the shell and average densities of $\sim 2,4$ and $4 \times 10^{4} \mathrm{~cm}^{-3}$. The maximum filling factor in this model (ratio of the length of our sight-line through the edge of the shell to that if seen faceon) is $\sim 3$ for a shell thickness $\sim 25$ per cent of the radius. These densities are consistent with those derived by applying PDR models to the observed line intensities.

Although the ability of UNSWIRF to resolve lines is 

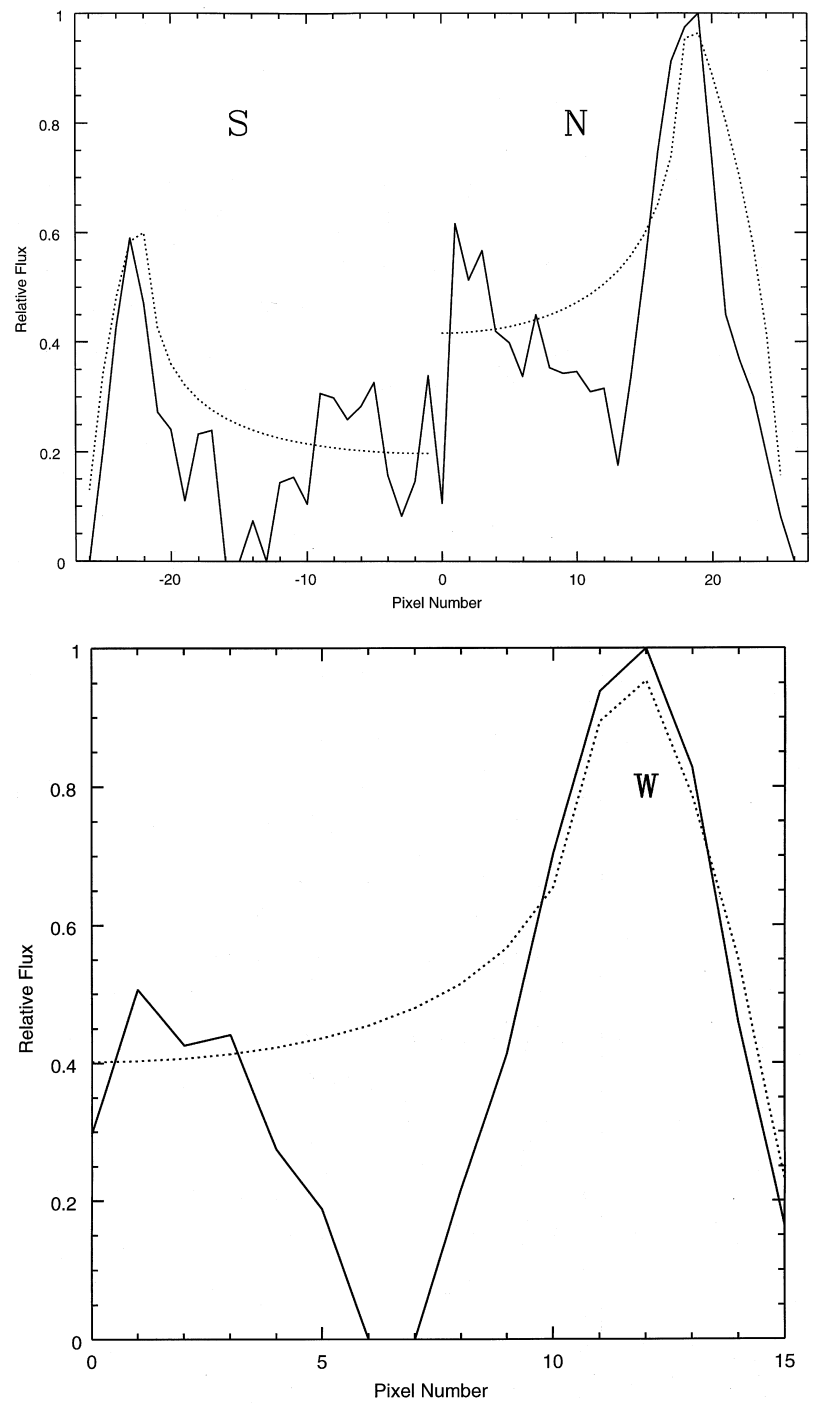

Figure 3. Cuts across the 2.12- $\mu \mathrm{m}$ emission in a line (top) northsouth through star 'A', and (bottom) east-west through star 'A' (solid lines), together with the best-fitting model results (dotted lines) for a spherical molecular shell irradiated by star 'A' with thickness 25 per cent of the shell radius, where dust extinction limits the region of $\mathrm{H}_{2}$ excitation to $A_{V}=1$. Star 'A' is at pixel $(0,0)$ in these plots, with 1 pixel $=1.2 \times 10^{16} \mathrm{~cm}$ for a distance to the source of $1.1 \mathrm{kpc}$.

limited by its instrumental profile width $\left(\sim 60-70 \mathrm{~km} \mathrm{~s}^{-1}\right.$, depending on parallelism), good signal-to-noise data such as the $2.12-\mu \mathrm{m}$ observations presented here allow us to measure changes in velocity over the image of only a few $\mathrm{km}$ $\mathrm{s}^{-1}$. The velocity data for the $2.12-\mu \mathrm{m}$ line centres (Table 1 ) show that motions in the gas must be small; differences of at most $10 \mathrm{~km} \mathrm{~s}^{-1}$ are seen between the different regions, with the exception of P 18-NW (Section 4.4).

\subsection{Line ratios}

\subsubsection{Observed parameter space}

For a dense PDR, where self-shielding is occurring, $\mathrm{H}_{2}$ exists at optical depths $A_{V}<1$ from the front surface of the

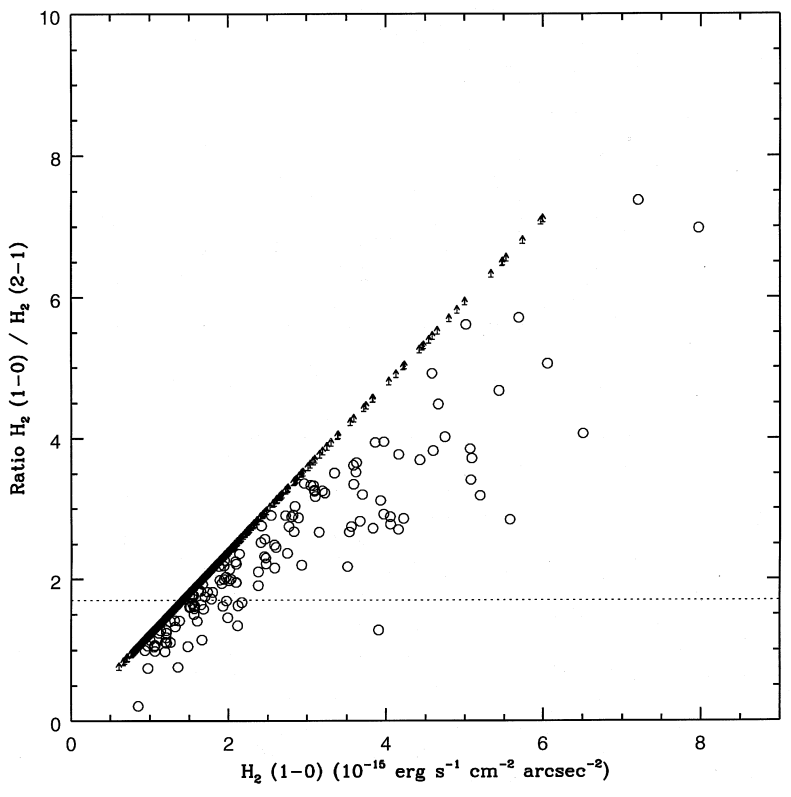

Figure 4. Plot of the $\mathrm{H}_{2}(1-0) /(2-1) \mathrm{S}(1)$ line ratio against 1-0 line flux in $\mathrm{P} 18$. Open circles are points which have $\mathrm{S} / \mathrm{N}>3$ in both lines, while the arrows denote points for which the $\mathrm{S} / \mathrm{N}$ of the $1-0$ line is $>3$, but the $\mathrm{S} / \mathrm{N}$ of the $2-1$ line is $<3$; thus these are lower limits in the ratio. The dotted horizontal line marks a ratio of 1.7 , the value expected for purely fluorescent emission. Both images were smoothed by a Gaussian function with $\sigma=0.3$ before the S/N criterion was applied.

cloud. Then, for a sufficiently strong far-UV radiation field, the gas temperature can reach $\sim 1000 \mathrm{~K}$ or higher, enabling collisions to redistribute the populations in the $\mathrm{H}_{2}$ energy levels. The $(1-0) /(2-1) S(1)$ line ratio then increases from the pure fluorescent value. It is sensitive to both the density and strength of the far-UV field, and so can be used to constrain these parameters in a source. This is different from shock-excited gas, where it has been shown empirically that there is little variation in this line ratio both among and within shocked sources (e.g. Burton et al. 1989). In particular, dense clumps of $\mathrm{H}_{2}$ inside the PDRs may have the $v=1$ level thermalized, while being surrounded by lower density gas exhibiting pure fluorescent line ratios. Such clumps would signal their presence by elevated levels of the $(1-0) /(2-1) S(1)$ ratio. This could then be used to probe the structure of the clouds.

We have thus sought to determine the fine structure in the spatial variation of the $(1-0) /(2-1) \mathrm{S}(1)$ ratio. We have registered the two images and applied a light Gaussian smoothe $(\sigma=0.3)$. In Fig. 4 we plot the line ratio so derived against the (1-0) $\mathrm{S}(1)$ line intensity. Two sets of symbols are shown in Fig. 4: open circles for data points where both the 1-0 and 2-1 $\mathrm{S}(1)$ lines have signal-to-noise ratios $\mathrm{S} / \mathrm{N}>3$, and lower limits for pixels where the $\mathrm{S} / \mathrm{N}$ for the $2-1 \mathrm{~S}(1)$ is $<3$ (calculated as though its flux were $3 \sigma$ ). The line ratio rises approximately linearly as the 1-0 $\mathrm{S}(1)$ line flux increases, to a maximum line ratio of $\sim 8$, but the dispersion in line ratio increases also. Similar plots for each of the eight distinct regions marked in Fig. 2 are presented in Fig. 5 (which also illustrates the effect of varying the far-UV 


\section{S. D. Ryder et al.}
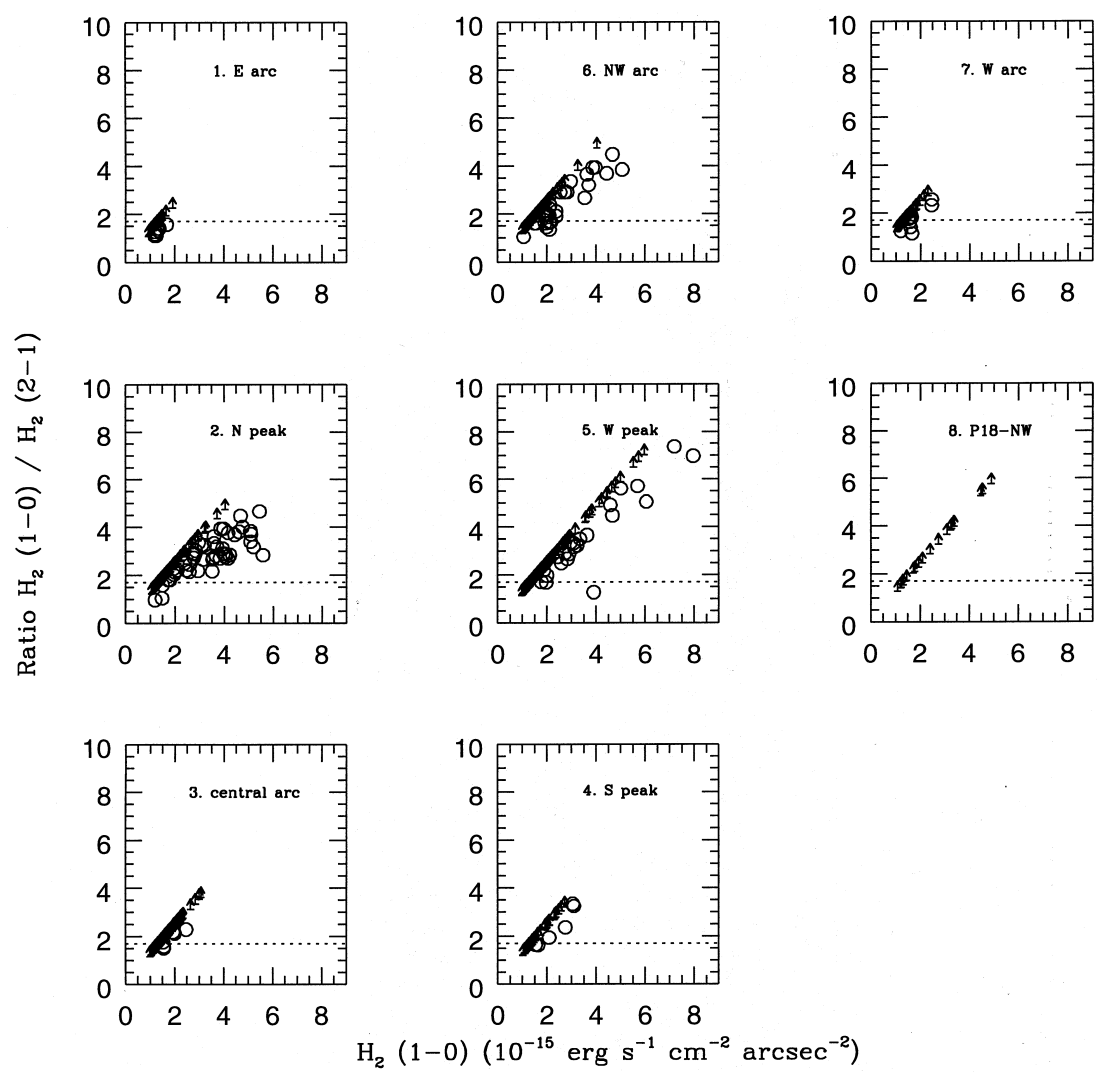

Figure 5. Same as Fig. 4, but for the specific regions of P 18 outlined in Fig. 2 and listed in Table 1.

field), and indicate that most of this spread in line ratio for a given 1-0 S(1) intensity is intrinsic to regions 2 and 6 (i.e. the northern regions of $\mathrm{P} 18$ ). Our interpretation of such trends must be guarded, however, owing to the large number of lower limits [non-detections in the $2-1 \mathrm{~S}(1)$ line]; high ratios for low 1-0 S(1) fluxes cannot be excluded by our data. Nevertheless, line ratios consistent with pure fluorescence become progressively rarer as the 1-0 S(1) line flux increases. We now seek to explain the apparent variations in line ratio with emission-line flux.

\subsubsection{Interpretation}

In pure fluorescent gas we would obtain a line ratio of $\sim 1.7$, independent of the line intensity. Localized regions of shocked gas would also be evident by a ratio of $\sim 10$ for a range of line intensities (see Brand et al. 1989). However, we see neither such behaviour in P 18 (with the exception of $\mathrm{P} 18-\mathrm{NW}$, which we argue in Section 4.4 to be physically unassociated with the other molecular hydrogen emission regions).

Qualitatively, the increase in line ratio can be understood as follows. At low density and far-UV fields, the $(1-0) /(2-1) S(1)$ ratio is purely fluorescent. However, for a fixed far-UV field, $\mathrm{H}_{2}$ of sufficiently high density will be selfshielded, producing a thermal contribution to the 1-0 S(1) line. Its flux will rise as the density does, while the 2-1 S(1) line remains purely fluorescent. Thus we expect both the strength of the 1-0 S(1) line and the ratio with the 2-1 S(1) line to rise as the density rises.

This line ratio variation can also be understood quantitatively through the PDR models of BHT. BHT derive a number of scaling relations depending on (i) whether $n / G_{0}$ is less than or greater than $40 \mathrm{~cm}^{-3}$ (determining if $\mathrm{H}_{2}$ selfshielding occurs), and (ii) whether $n$ is less than or greater than $n_{\text {crit }}$, the density where collisional de-excitation equals the radiative decay rate from a level. The line intensity can be divided into a UV-pumped $\left(I_{\text {pump }}\right)$ and a thermal $\left(I_{\text {thermal }}\right)$ part, as follows:

When $n / G_{0} \preceq 40 \mathrm{~cm}^{-3}$ (self-shielding unimportant):

$I_{\text {pump }} \propto \begin{cases}n & \left(n<n_{\text {crit }}\right) \\ n_{\text {crit }} & \left(n>n_{\text {crit }}\right) .\end{cases}$

When $n / G_{0} \geq 40 \mathrm{~cm}^{-3}$ (self-shielding important):

$I_{\text {pump }} \propto \begin{cases}G_{0} & \left(n<n_{\text {crit }}\right) \\ G_{0} n_{\text {crit }} / n & \left(n>n_{\text {crit }}\right) .\end{cases}$

The thermal contribution is given by

$I_{\text {thermal }} \propto \begin{cases}n N_{2} \gamma & \left(n<n_{\text {crit }}\right) \\ N_{2} \mathrm{e}^{-E / k T} / Z(T) & \left(n>n_{\text {crit }}\right),\end{cases}$ 
where $N_{2}$ is the column of warm $\mathrm{H}_{2}$ (i.e. at $A_{V}<1$ ) with temperature $\sim T, E$ is the energy of the level of interest, $\gamma$ is the collisional excitation rate from the ground state, and $Z(T)$ is the $\mathrm{H}_{2}$ partition function.

For the bulk of the gas, with density $n \sim(2-4) \times 10^{4} \mathrm{~cm}^{-3}$ and $G_{0} \sim 10^{3-4}$, we have both $n<n_{\text {crit }}$ and $n / G_{0}<40 \mathrm{~cm}^{-3}$. The line intensity is then simply proportional to the product of the density and the filling factor, $f$. To within a factor of 3 , for $n \prec 10^{5} \mathrm{~cm}^{-3}, I_{1-0 \mathrm{~S}(1)} \sim 3 f n_{4} \times 10^{-16} \mathrm{erg} \mathrm{cm}^{-2} \mathrm{~s}^{-1}$ arc$\sec ^{-2}$, where $n_{4}$ is the density in units of $10^{4} \mathrm{~cm}^{-3}$ (see equation 1). As discussed in Section 4.1, this is consistent with the average line fluxes observed for filling factors of $1-3$, with $n_{4}=4$, the value deduced from the geometrical model (see Section 4.2).

The detailed models of BHT show that the thermal contribution to the line intensities is neligible when $G_{0}=10^{3}$, and also when $n \leq 10^{5} \mathrm{~cm}^{-3}$ for $G_{0}=10^{4}$. However, for the larger far-UV field, the thermal contribution to the 1-0 S(1) line flux is $\sim 70$ per cent for $n=10^{6-7} \mathrm{~cm}^{-3}$ [the 2-1 S(1) line remains purely fluorescent]. Thus higher line fluxes and ratios $>2$ imply the presence of some gas of at least this density.

For the east and west arcs (see Table 1), where the $G_{0}$ is $\sim 2000$, the line ratio is constant at $\sim 1.7$. In the north and south peaks and the north-west arc, with $G_{0}=5060,3500$ and 3200 respectively, a thermal contribution to the line ratio is becoming apparent. At the west peak, closest to the illuminating source, where $G_{0}=17800$, both the 1-0 S(1) ine intensity and $1-0 / 2-1 S(1)$ ratio are highest; the $2-1$ $\mathrm{S}$ (1) line flux density is, however, relatively diffuse at this latter position. On the basis of Fig. 5, it would seem reasonable that the central arc, though apparently superimposed on star 'A', must in fact lie at a similar distance from it as the east and west arcs.

These observations can be understood as follows. For the arcs, where the far-UV field is low, the emission is purely fluorescent, but the line ratio is insensitive to density. At the north and south peaks, a thermal contribution would be expected for $n \geq 10^{6} \mathrm{~cm}^{-3}$. The line ratios at the brightest pixels suggest that $\sim 30$ per cent of the $1-0 \mathrm{~S}(1)$ emission there is thermal, and thus has densities this high. At the west peak, self-shielding will occur when $n>40 G_{0} \sim 5 \times 10^{5} \mathrm{~cm}^{-3}$. The data here are thus sensitive to the presence of gas denser than this, a density also larger than $n_{\text {crit }}$. Ratios observed are as high as 7 , implying that as much as 70 per cent of the emitting gas at those positions is at least this dense.

Over the west peak, the specific intensity of the 2-1 S(1) line is relatively weaker, in comparison with that of the $1-0$ $\mathrm{S}$ (1) line, than at the other peaks excited by star 'A'. In this region, the far-UV field $G_{0}$ exceeds $10^{4}$. There is a significant thermal contribution to the $1-0 \mathrm{~S}(1)$ flux from dense gas. The 2-1 S(1) flux, however, remains purely fluorescent. Indeed, as seen by equation (2), its intensity is predicted to decrease as $1 / n$, consistent with the observation that it gets relatively weaker. At other peaks, the far-UV field is not sufficiently high for us to expect to observe this behaviour.

The maximum 1-0 S(1) line intensity that is purely fluorescent, arising from gas of average cloud density, occurs when the filling factor is $\sim 3$, and the flux density is $\sim 4 \times 10^{-16} \mathrm{erg} \mathrm{cm}^{-2} \mathrm{~s}^{-1} \operatorname{arcsec}^{-2}$ for the spherical shell geometry we have modelled. For higher line fluxes a ther- mal component is present, and the line ratio rises above the pure fluorescent value. The thermal component arises from gas of density at least $10^{6} \mathrm{~cm}^{-3}$, and both the highest line fluxes and line ratios occur when the filling factor of this dense gas is greatest.

The spread of observed line ratios between the minimum and maximum values for a fixed 1-0 S(1) line flux can be understood by a variation of the relative filling factors for the low-density and high-density gas. The lowest ratio occurs when the proportion of low-density gas is highest, and rises, for a fixed flux level, as both the fraction of lowdensity gas falls and that of the high-density gas rises. Thus data with sufficiently high $\mathrm{S} / \mathrm{N}$ to map the line ratio variation accurately from pixel to pixel could be used to determine the microstructure of the clumping. The line ratio map (Fig. 2), for positions where $G_{0} \geq 3000$, is effectively showing the relative amounts of high- and low-density gas.

We thus conclude that the variations in the line ratio are consistent with the PDR models, self-shielding of gas occurring in its densest parts, with thermal contributions evident when exposed to the highest radiation fields. Localized regions where the density is more than 10 times the average exist throughout the molecular cloud. The highest line fluxes and line ratios occur together, where both the filling factor of low- and high-density gas is greatest, and changes in line ratio from position to position reflect changes in the relative filling factors of these components.

\section{$4.4 \quad \mathrm{P} 18-\mathrm{NW}$}

The isolated flux peak to the north-west (designated by us as P 18-NW), however, shows no evidence for fluorescent line emission. The 2-1 S(1) line here is barely detected, and the $1-0 / 2-1 \mathrm{~S}(1)$ line ratio is clearly thermal in nature, similar to shock-excited values. $\mathrm{P} 18-\mathrm{NW}$ is too far from star ' $\mathrm{A}$ ' for collisional fluorescence to be significant, as $G_{0}<500$. We suggest that there is a localized source of heating, possibly shocking the gas.

The simultaneous velocity coverage of UNSWIRF offers at least one clue. We notice that the line-centre velocity in $\mathrm{P} 18-\mathrm{NW}$ is also quite different from the rest of $\mathrm{P} 18$. On account of its redshift of $+14 \mathrm{~km} \mathrm{~s}^{-1}$ relative to the central arc, we speculate that $\mathrm{P} 18-\mathrm{NW}$ might, in fact, be associated in some way with the $\mathrm{CO}$ outflow source observed by Fukui (1989) and by Felli, Palagi \& Tofani (1992). Although Fukui identified the outflow with the position of star 'A', the 3-arcmin beam used for the $\mathrm{CO}$ observations is much larger than the 45-arcsec separation between star 'A' and P 18-NW. The IRAS point source discussed by López et al. (1988) and by Felli et al. (1992), namely IRAS $06572-0742$, has a bolometric luminosity $\sim 1500 \mathrm{~L}_{\odot}$ and colours suggestive of an ultracompact $\mathrm{H}$ II region, although neither $\mathrm{H}_{2} \mathrm{O}$ (Felli et al. 1992) nor methanol (Walsh et al. 1997) masers have been detected from it. While the IRAS positional uncertainties do not preclude an association of $\mathrm{P} 18-\mathrm{NW}$ with the IRAS source, it is perhaps more likely that the $\mathrm{H}_{2}$ line emission observed from P 18-NW is shock-excited by the interaction of an outflow, from an embedded source in $\mathrm{P} 18$, with the ambient cloud material. More detailed $\mathrm{CO}$ observations are needed to clarify the influence of any outflow activity on the excitation and environment of $\mathrm{P} 18$. 


\section{CONCLUSIONS}

The reflection nebula Parsamyan 18 has been imaged in both the 2.122- and 2.248- $\mu$ m emission lines of molecular hydrogen using the UNSW Infrared Fabry-Perot on the AAT, allowing us to study variations in their ratio within the nebula. The emission-line morphology appears as a pair of overlapping arcs, consistent with incomplete PDR shells irradiated by a single, early B-type star. We believe that we can identify this star in our continuum maps, which contradicts previous claims that the stellar-like sources in $\mathrm{P} 18$ are 'windows' in a circumstellar dust shell, illuminated by a compact $\mathrm{H}$ in region. Modelling suggests that the thickness of the fluorescing shells is roughly 25 per cent of their radius, with average gas densities of $(2-4) \times 10^{4} \mathrm{~cm}^{-3}$, and dust extinction limiting the penetration of the far-UV photons. In addition, some regions of $\mathrm{P} 18$ display line ratios indicative of collisional fluorescence in the densest regions exposed to the highest radiation fields. In general, those regions with the highest intrinsic 1-O S(1) intensities also have the highest (1-0)/(2-1) $S(1)$ line ratios, a result of this process. Within these regions, large ranges in the observed line ratio can be understood as arising from the underlying variations in the relative filling factors of high- and low-density gas. We have discovered an isolated knot of shock-excited $\mathrm{H}_{2}$ gas just outside the nebula, which we argue on kinematical grounds is more likely to be associated with the $\mathrm{CO}$ outflow source reported in this region. This study highlights the advantages of an imaging Fabry-Perot system over traditional long-slit spectroscopy for emission-line imaging, particularly for understanding the excitation mechanism of molecular hydrogen.

\section{ACKNOWLEDGMENTS}

We acknowledge useful discussions with Peter Brand and Antonio Chrysostomou, and suggestions from the referee for improving the paper. We thank Yin-Sheng Sun for valu- able assistance in calibrating UNSWIRF. SDR acknowledges the receipt of a UNSW Vice-Chancellor's Post-doctoral Fellowship. UNSWIRF was funded by a grant from the Australian Research Council.

\section{REFERENCES}

Allen D. A. et al., 1993, Proc. Astron. Soc. Aust., 10, 298

Black J. H., Dalgarno A., 1976, ApJ, 203, 132

Black J. H., van Dishoeck E. F., 1987, ApJ, 322, 412

Brand P. W. J. L., Toner M. P., Geballe T. R., Webster A. S., Williams P. M., Burton M. G., 1989, MNRAS, 236, 929

Burton M. G., Brand P. W. J. L., Geballe T. R., Webster A. S., 1989, MNRAS, 236, 409

Burton M. G., Geballe T. R., Brand P. W. J. L., Moorhouse A., 1990, ApJ, 352, 625

Burton M. G., Hollenbach D. J., Tielens A. G. G. M., 1990, ApJ, 365,620 (BHT)

Cohen M., 1974, PASP, 86, 813

Felli M., Palagi F., Tofani G., 1992, A\&A, 255, 293

Fukui Y., 1989, in Reipurth B., ed., Low Mass Star Formation and Pre-Main Sequence Objects. ESO, Garching, p. 95

Gatley I. et al., 1987, ApJ, 318, L73

Habing H. J., 1968, Bull. Astron. Inst. Netherlands, 19, 421

Hayashi M., Hasegawa T., Gatley I., Garden R., Kaifu N., 1985, MNRAS, 215, 31P

Hilton J., Lahulla J. F., 1995, A\&AS, 113, 325

Hodapp K.-W., 1994, ApJS, 94, 615

López J. A., Roth M., Friedman S. D., Rodríguez L. F., 1988, Rev. Mex. Astron. Astrofis., 16, 99

Sellgren K., 1986, ApJ, 305, 399

Sellgren K., Werner M. W., Allamandola L. J., 1996, ApJS, 102, 369

Sternberg A., Dalgarno A., 1989, ApJ, 338, 197

Stutzki J., Stacey G. J., Genzel R., Graf U. U., Harris A. I., Jaffe D. T., Lugten J. B., Poglitsch A., 1990, in Watt G. D., Webster A. S., eds, Submillimetre Astronomy. Kluwer, Dordrecht, p. 269

Tielens A. G. G. M., Hollenbach D. J., 1985, ApJ, 291, 722

Walsh A. J., Hyland A. R., Robinson G., Burton M. G., 1997, MNRAS, 291, 261 